\title{
Excited States and Collectivity in ${ }^{88}$ Se
}

\author{
G. Simpson 1,*, I. Grachev ${ }^{1,2}$, G. Thiamova ${ }^{1}$, M. Ramdhane ${ }^{1}$, K. Sieja ${ }^{3}$, A. Blanc ${ }^{2}$, M. Jentschel ${ }^{2}$, \\ U. Köster ${ }^{2}$, P. Mutti ${ }^{2}$, T. Soldner ${ }^{2}$, G. de France ${ }^{4}$, C. A. Ur ${ }^{5,6}$, and W. Urban ${ }^{7}$ \\ ${ }^{1}$ Laboratoire de Physique Subatomique et de Cosmologie, Université Grenoble Alpes, CNRS/IN2P3, Greno- \\ ble INP, F-38026 Grenoble, France \\ ${ }^{2}$ Institut Laue-Langevin, 71 avenue des Martyrs, 38042 Grenoble Cedex 9, France \\ ${ }^{3}$ Université de Strasbourg, IPHC, 23 rue du Loess 67037 Strasbourg, CNRS, UMR7178, 67037 Strasbourg, \\ France \\ ${ }^{4}$ Grand Accélérateur National d'lons Lourds, CEA/DRF-CNRS/IN2P3, Boulevard Henri Becquerel, F-14076 \\ Caen, France \\ ${ }^{5}$ INFN, via Marzolo 8, 35131 Padova, Italy \\ ${ }^{6}$ ELI-NP/IFIN-HH, 30 Reactorului Str., 077125 Magurele, Romania \\ ${ }^{7}$ Faculty of Physics University of Warsaw, ulica Pasteura 5, PL-02-093 Warsaw, Poland
}

\begin{abstract}
The $\gamma$ decays of excited states in the very neutron-rich nucleus ${ }^{88}$ Se have been observed following the cold neutron-induced fission of ${ }^{235} \mathrm{U}$ at the PF1B facility of the Institut Laue-Langevin (ILL), Grenoble. The EXILL array was used to measure $\gamma-\gamma-\gamma$ coincidences, which were then analysed to build a level scheme of ${ }^{88} \mathrm{Se}$. A low $\left(2_{1}^{+}\right)$ energy hints at the onset of quadrupole deformation and the identification of possible members of a $\left(2_{2}^{+}\right)$band provide evidence for $\gamma$ vibrations. Shell-model calculations using a ${ }^{78} \mathrm{Ni}$ core reproduce the experimental decay scheme well, implying that interactions between particles in the $\pi f_{5 / 2} p$ and $v s d$ orbits are responsible for much of the collectivity present. The algebraic collective model has also been used to interpret the experimental data, showing that that the experimental spectrum is consistent with ${ }^{88} \mathrm{Se}$ being a transitional nucleus, possessing weak static $\beta$ deformation and $\gamma$ instability.
\end{abstract}

\section{Introduction}

A key question in nuclear-structure studies is how to microscopically describe the onset of collective behavior when moving away from shell closures in terms of the interactions between individual nucleons occupying single-particle states. Little is known about the neutron-rich $A \sim 90$ nuclei North-East of ${ }^{78} \mathrm{Ni}$ which can possess collective states when just 4 valence protons and 2 neutrons are present [1]. The fragile nature of the ${ }^{78} \mathrm{Ni}$ core has been suggested as a likely reason for the presence of $\left(5,6^{+}\right)$ states at an energy of $\sim 3 \mathrm{MeV}$ in ${ }^{82} \mathrm{Ge}[2]$ and a tendency towards $\gamma$ softness in ${ }^{84} \mathrm{Ge}$ [1]. Experimental data suggest the $\left(2_{1}^{+}\right)$state of ${ }^{86} \mathrm{Ge}$ may be deformed [3]. Recently evidence has been found for collective behavior in the neutron-rich Se isotopes, namely the beginning of a $\gamma$-band in ${ }^{86} \mathrm{Se}[4,5]$, and a $j-1$ ground-state spin of $\left(3 / 2^{+}\right)$for ${ }^{87} \mathrm{Se}[6]$. An $886.2-\mathrm{keV}$ transition was assigned as the decay

*e-mail: simpson@1psc.in2p3.fr 


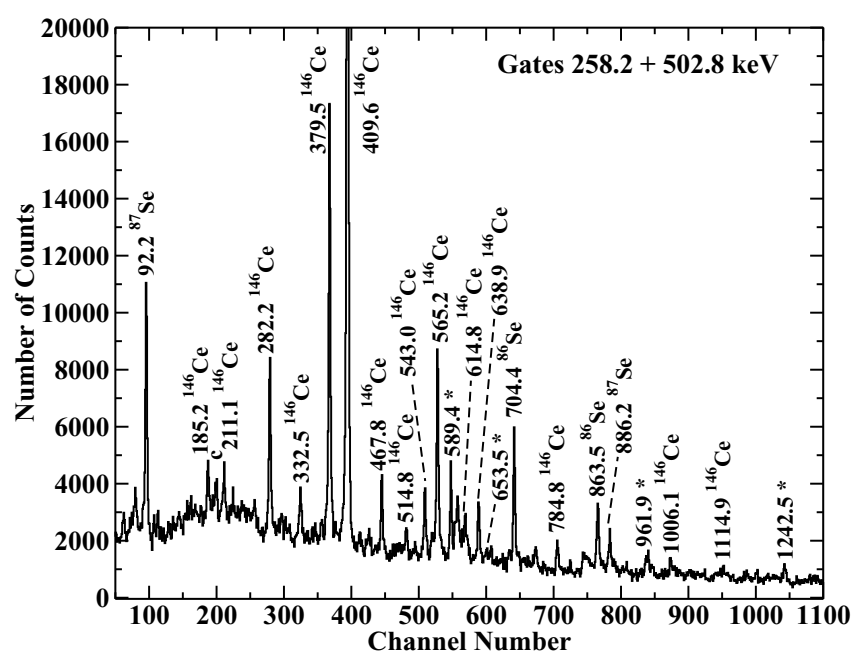

(a)

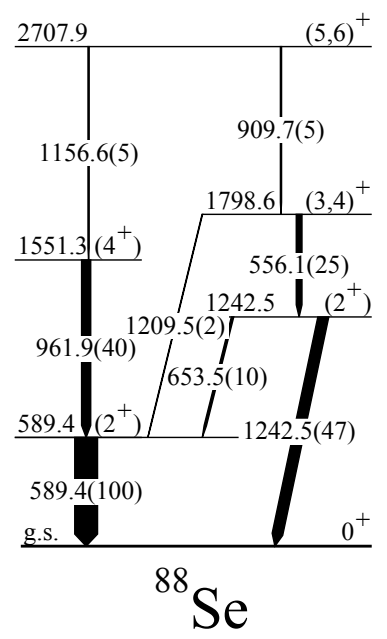

(b)

Figure 1. (a) A $\gamma$-ray coincidence spectra obtained by double gating on the 258.2 and $502.8 \mathrm{keV} \gamma$-rays transitions in ${ }^{146} \mathrm{Ce}$. New lines of interest are labeled with * and contaminant transitions with c. (b) Level scheme of ${ }^{88} \mathrm{Se}$ derived in the present work.

of the $\left(2_{1}^{+}\right)$state in ${ }^{88} \mathrm{Se}[7]$ however this was recently attributed to ${ }^{87} \mathrm{Se}$ [6], leaving the level scheme of ${ }^{88} \mathrm{Se}$ unknown.

Comprehensive shell-model (SM) and symmetry conserved configuration mixing-Gogny (SCCM) calculations have been published for ${ }^{88} \mathrm{Se}$ [8]. These predict that a significant amount of both quadrupole deformation and triaxiality should be present in ${ }^{88} \mathrm{Se}$ [8]. In this context, it is interesting to study the structure of ${ }^{88} \mathrm{Se}$.

The neutron-rich $A \sim 90$ Se nuclei are populated with a higher fission yield in the ${ }^{235} \mathrm{U}\left(n_{t h}, f\right)$ reaction than following the spontaneous fission of ${ }^{248} \mathrm{Cm}$ or ${ }^{252} \mathrm{C}$, used in previous prompt- $\gamma$ studies of this isotopic chain $[4,6,7]$. The ${ }^{235} \mathrm{U}\left(n_{t h}, f\right)$ reaction has therefore been used, in conjunction with the EXILL Ge array [9], to study $\gamma$-rays emitted by ${ }^{88} \mathrm{Se}$.

\section{Experimental Technique}

The experiment was carried out at the PF1B cold-neutron beam of the Institute Laue-Langevin in Grenoble, using the EXILL array of Ge detectors [9]. Thermal neutron-induced fission of a ${ }^{235} \mathrm{U}$ target was used to create ${ }^{88}$ Se nuclei and prompt $\gamma-\gamma-\gamma$ spectroscopy allowed decays from their excited states to be observed. More details on the experimental technique can be found in Ref. [10].

\section{Experimental Results}

Gates were set on known transitions from each of the isotopes ${ }^{84-87} \mathrm{Se}$ and the distribution of the average masses of the Ce partner isotopes obtained. From this it was found that ${ }^{146} \mathrm{Ce}$ is the most likely fission partner of ${ }^{88} \mathrm{Se}$ in the ${ }^{235} \mathrm{U}\left(n_{t h}, f\right)$ reaction. As no transitions are known in ${ }^{88} \mathrm{Se}$ then gates were set on the intense $2^{+} \rightarrow 0^{+}(258.2 \mathrm{keV})$ and $6^{+} \rightarrow 4^{+}(502.8 \mathrm{keV})$ transitions of ${ }^{146} \mathrm{Ce}$ producing 

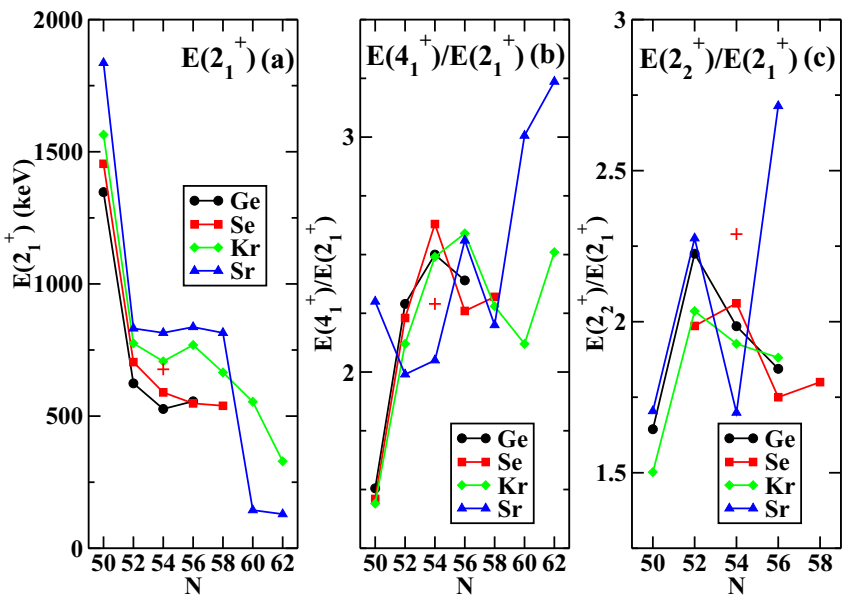

Figure 2. (a) Experimental energies of the $\left(2_{1}^{+}\right)$states, (b) ratios of the level energies $E\left(4_{1}^{+}\right) / E\left(2_{1}^{+}\right)$and (c) $E\left(2_{2}^{+}\right) / E\left(2_{1}^{+}\right)$of $50 \leq N \leq 58 \mathrm{Ge}, \mathrm{Se}, \mathrm{Kr}$, and $\mathrm{Sr}$ isotopes. Shell-model calculated values for ${ }^{88} \mathrm{Se}$ are highlighted by a + symbol.

the spectrum shown in Fig. 1. Here one observes lines previously attributed to ${ }^{86} \mathrm{Se}$ (4n channel) [4] and ${ }^{87} \mathrm{Se}$ (3n channel) [6], along with other transitions from ${ }^{146} \mathrm{Ce}$ [11]. Four unidentified lines are present at $589.4,653.5,961.9$, and $1242.5 \mathrm{keV}$.

A double gate was then set on the new 589.4- and 961.9-keV lines and strong $\gamma$-ray transitions from the complementary fragments ${ }^{145,146,147} \mathrm{Ce}$ were present. From the measured average mass of the coincident $\mathrm{Ce}$ isotopes [145.8(2)] this cascade was assigned to ${ }^{88} \mathrm{Se}$. The above coincidence relationships, and further gating, has allowed the decay scheme of ${ }^{88} \mathrm{Se}$ shown in Fig. 1(b) to be constructed. This analysis is explained in more detail in Ref. [10] along with justifications for the proposed spin assignments.

\section{Discussion}

The systematic evolution of the experimental energies of the known $\left(2_{1}^{+}\right)$states in the $50 \leq N \leq 58$ $\mathrm{Ge}, \mathrm{Se}, \mathrm{Kr}$ and $\mathrm{Sr}$ isotopes [12-15] are shown in Fig. 2. Here one can see that the $E\left(2_{1}^{+}\right)$values of each isotonic chain between $N=52-58$ decrease when going from $\mathrm{Sr}$ down to $\mathrm{Ge}$, implying an increase in quadrupole deformation. The $E\left(4_{1}^{+}\right) / E\left(2_{1}^{+}\right)$energy ratios of the Se and $\mathrm{Kr}$ nuclei increase from 2.12.3 at $N=52$, which are typical values for vibrational excitations, to 2.5-2.6 at $N=54$, characteristic of $\gamma$-unstable or shape transitional nuclei. From their similar $E\left(2_{1}^{+}\right)$values, and $E\left(4_{1}^{+}\right) / E\left(2_{1}^{+}\right)$ratios, it is likely that the low-energy states of ${ }^{86,88}$ Se have comparable collective characteristics.

Raman's empirical formula, which relates $\beta$ to $E\left(2_{1}^{+}\right)$and mass [16], allows $\beta=0.22(2)$ to be estimated for ${ }^{88} \mathrm{Se}$. This is larger than $\beta=0.17$ calculated by the FRDM model [17], possibly pointing to a faster onset of deformation than expected. It is also worth noting that the $E\left(4_{1}^{+}\right) / E\left(2_{1}^{+}\right)$ratio for ${ }^{88} \mathrm{Se}$ is the highest one so far reported in this region for nuclei $N<60$, pointing to increased rigidity.

The $\left(2_{2}^{+}\right)$level energy of ${ }^{88} \mathrm{Se}$ is one of the lowest known in the region. It lies below the $\left(4_{1}^{+}\right)$state, indicating triaxial softness. However the $E\left(2_{2}^{+}\right) / E\left(2_{1}^{+}\right)$ratios presented in Fig. 2(c) show that several other nuclei in the region appear to be softer towards triaxial deformation. 


\subsection{Shell-Model Interpretation}

Shell Model

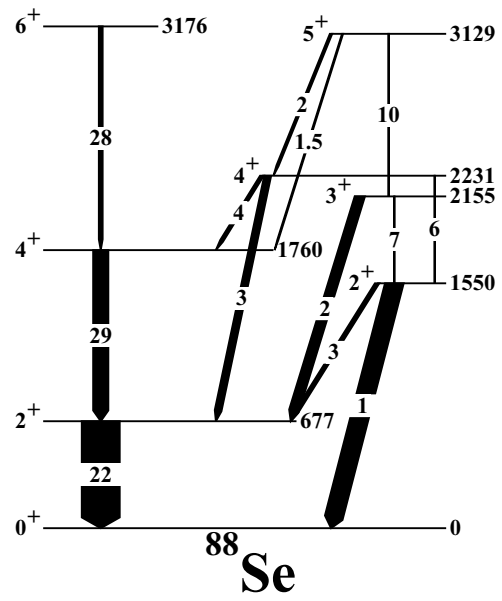

Exp.

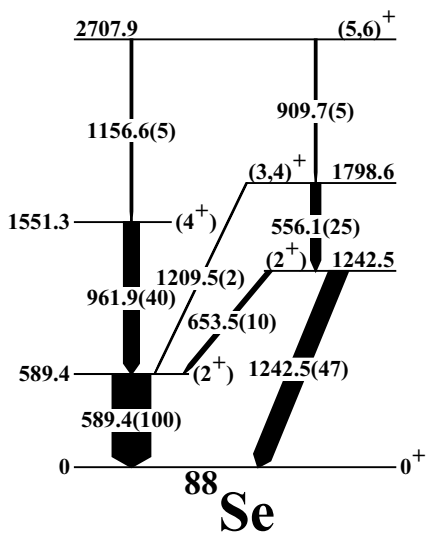

Figure 3. Calculated and experimental energies of excited states in ${ }^{88} \mathrm{Se}$. Line widths represent transition intensities, including any M1 and E2 components. $B(E 2)$ values in W.u. are labeled on the arrows. See text for more details.

To further investigate the properties of ${ }^{88} \mathrm{Se}$ the experimental level scheme has been compared to the results of a $S M$ calculation displayed in Fig. 3. The valence space used is comprised of the $\pi\left(f_{5 / 2}, p_{3 / 2}, p_{1 / 2}, g_{9 / 2}\right)$ and $v\left(d_{5 / 2}, d_{3 / 2}, s_{1 / 2}, g_{7 / 2}, h_{11 / 2}\right)$ orbits. A recent effective interaction for this model space (Ni78-II) has been employed (see [10] and references therein).

The general features of the experimental level scheme of ${ }^{88} \mathrm{Se}$ are reproduced reasonably well by the calculation, including the $E\left(4_{1}^{+}\right) / E\left(2_{1}^{+}\right)$and $E\left(2_{2}^{+}\right) / E\left(2_{1}^{+}\right)$ratios, which are marked by crosses in Figs. 2(b) and (c), though some collectivity is missing. The agreement between the experimental and calculated $E\left(2_{1}^{+}\right), E\left(2_{2}^{+}\right)$, and $E\left(4_{1}^{+}\right)$values is within the typical errors, though this worsens for higher energy states. Figure 3 also shows the predicted transition intensities as arrow widths and these include any contributions from both E2 and M1 multipolarities. The sum of the arrow widths out of each theoretical level is the same as that of its experimental counterpart and the agreement is mostly good. Effective charges of $e_{\pi}=1.7 e$ and $e_{v}=0.7 e$ were used, the same as in previous works using both the Ni78-I and Ni78-II interactions [8, 18]. These high effective charges indicate that orbits from outside the valence space also contribute to the collectivity present in ${ }^{88} \mathrm{Se}$. Applying Kumar's formula [19] to the $S M B(E 2)$ values allows the intrinsic shape parameter $\beta=0.23$ to be obtained for the ground state, consistent with empirically derived one. The calculated level sequence, with a doublet of $3^{+}, 4_{2}^{+}$states, is characteristic of a $\gamma$-unstable nucleus. This feature, however, is not confirmed in the present experimental scheme, due to fission favoring the population of yrast states.

When moving away from the $Z=28$ and $N=50$ shell closures the $\pi f_{5 / 2}, \pi p_{3 / 2}$ and $v d_{5 / 2}, v s_{1 / 2}$ orbits are the first to be filled. The proximity of the $\pi p_{1 / 2}$ and $v d_{3 / 2}, v g_{7 / 2}$ orbits would lead to two pseudo-SU(3) blocks being formed, as considered in Ref. [8], and shown to be consistent with the $S M$ diagonalization results for ${ }^{88} \mathrm{Se}[8,10]$. An inspection of the $S M$ wave functions shows that the $\pi p f$ and $v s d$ orbits are dominant and seem to be responsible for much of the collectivity present in ${ }^{88} \mathrm{Se}$. These conclusions are in line with recent results on the neighboring nuclei ${ }^{87} \mathrm{Se}$ and ${ }^{88} \mathrm{Br}$ where $S M$ 


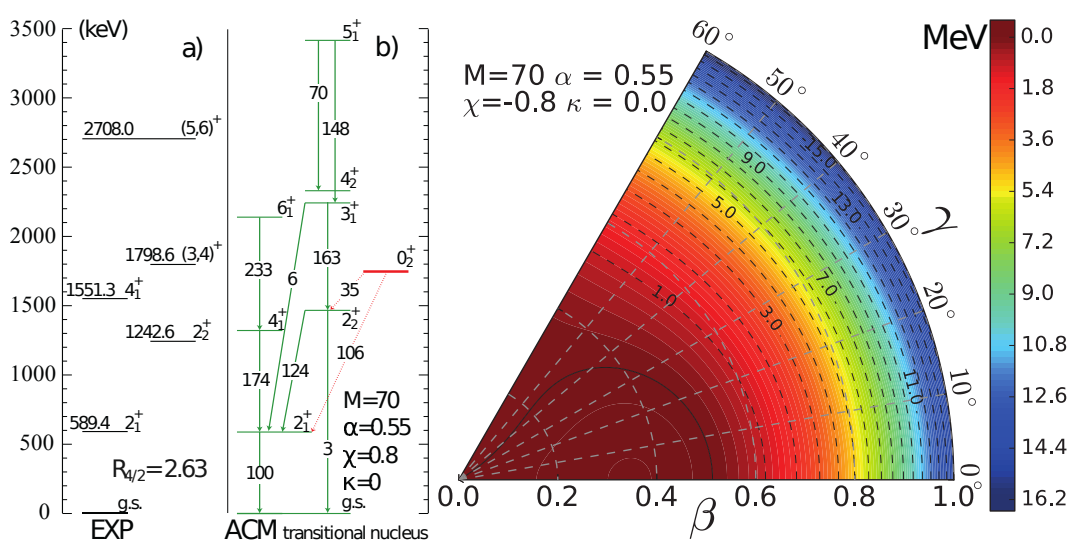

(a)

(b)

Figure 4. (a) Level scheme and (b) potential energy surface obtained using the ACM with parameters for a transitional nucleus of $M=70, \alpha=0.55, \chi=-0.8$, and $\kappa=0$. Calculated relative reduced $E 2$ transition rates are also labelled on the arrows connecting the levels. These transition strengths are normalised to the $2_{1}^{+} \rightarrow 0_{1}^{+}$ one.

calculations have shown that the collectivity present is due to the $v d_{5 / 2}^{3}$ configuration $[6,20]$, and in particular its coupling to $\pi 2^{+}$states.

\subsection{ACM Interpretation}

The level scheme of ${ }^{88} \mathrm{Se}$ obtained in the present work has been interpreted within the framework of the Algebraic Collective Model (ACM). The ACM is the algebraic version of Bohr's collective model and its Hamiltonian, in terms of the deformation parameters $\beta$ and $\gamma$, is

$$
H(M, \alpha, \kappa, \chi)=-\frac{\nabla^{2}}{2 M}+\frac{1}{2} M\left[(1-2 \alpha) \beta^{2}+\alpha \beta^{4}\right]-\chi \beta \cos 3 \gamma+\kappa \cos ^{2} 3 \gamma
$$

where $M$ is the effective mass and

$$
\nabla^{2}=\frac{1}{\beta^{4}} \frac{\partial}{\partial \beta} \beta^{4} \frac{\partial}{\partial \beta}-\frac{1}{\beta^{2}} \Lambda^{2}
$$

with $\Lambda$ the Casimir operator [21]. The term $\cos ^{2} 3 \gamma$ allows the generation of a triaxial minimum in the potential energy surface, with $\kappa$ determining the degree of deformation. This Hamiltonian is capable of describing a wide range of collective spectra and is also applicable in regions undergoing phase transitions from axial to triaxial deformation, or from spherical to deformed, where $\alpha$ is the control parameter.

The number of free parameters in the ACM means that multiple solutions are possible for ${ }^{88} \mathrm{Se}$. The level energies of ${ }^{88} \mathrm{Se}$ were reproduced when this nucleus is described as either a $\gamma$-unstable or a transitional nucleus. Although the former description reproduces better the level energies the $\gamma$-branching ratios are far from the experimental ones. The opposite is true for a transitional picture.

For a transitional description of ${ }^{88}$ Se parameters of $M=70, \alpha=0.55, \chi=-0.8$, and $\kappa=0$ were used. The value of $\alpha \sim 0.5$ is typical for a transitional nucleus undergoing a phase change from a 
spherical shape to an axially deformed one. As $\alpha>0.5$ this implies non-zero static $\beta$ deformation. A wide, shallow minimum is present in the potential energy surface of Fig. 4(b) at values of $\beta=0$ to 0.3 . Although a small minimum is present at $\gamma=0^{\circ}$, practically this nucleus can be described as $\gamma$ unstable.

The calculated relative reduced transition rates within the ground-state band are in fair agreement with those obtained using the $S M$, shown in Fig. 3. However, with the exception of the $2_{2}^{+} \rightarrow 0_{1}^{+}$ transition, decays within the $\gamma$ band, and out of it, are predicted to be much stronger. For example the predicted $B\left(E 2 ; 2_{2}^{+} \rightarrow 2_{1}^{+}\right) / B\left(E 2 ; 2_{2}^{+} \rightarrow 0_{1}^{+}\right)$is much higher than the one obtained from the measured transition intensities. As this ratio changes rapidly as a function of $\alpha$ here, then a small increase in this parameter allows the experimental $B\left(E 2 ; 2_{2}^{+} \rightarrow 2_{1}^{+}\right) / B\left(E 2 ; 2_{2}^{+} \rightarrow 0_{1}^{+}\right)$value to be reproduced. Further experimental measurements are necessary to definitively classify the nature of this nucleus.

\section{Conclusion}

Prompt $\gamma-\gamma-\gamma$ coincidence measurements performed using the EXILL Ge array, following the coldneutron induced fission of a ${ }^{235} \mathrm{U}$ target, have allowed a first level scheme of ${ }^{88} \mathrm{Se}$ to be established. The energies of the $\left(2_{1}^{+}\right)$and $\left(4_{1}^{+}\right)$states are characteristic of a $\gamma$-unstable or transitional nucleus and hint at an increase in collectivity compared to ${ }^{86} \mathrm{Se}$. The identification of a low-lying $\left(2_{2}^{+}\right)$level indicates the presence of $\gamma$ vibrations in this nucleus. Shell-model predictions were found to reproduce many of the general properties of the ${ }^{88} \mathrm{Se}$ level scheme and show that the occupation of the $\pi f_{5 / 2} p$ and $v s d$ orbits is mostly responsible for the collectivity present. Calculations performed using the $\mathrm{ACM}$ also reproduce the decay scheme of ${ }^{88} \mathrm{Se}$ and allow the structure of this nucleus to be classified as transitional, in line with the systematics. The ACM also predicts that ${ }^{88} \mathrm{Se}$ has non-zero static $\beta$ deformation and is $\gamma$-unstable.

\section{Acknowledgements}

The EXILL campaign would not have been possible without the support of several services at the ILL, GANIL and the LPSC. The EXOGAM collaboration and the INFN Legnaro are acknowledged for the loan of Ge detectors.

\section{References}

[1] M. Lebois et al., Phys. Rev. C 80, 044308 (2009)

[2] T. Rząca-Urban, W. Urban, J.L. Durell, A.G. Smith, I. Ahmad, Phys. Rev. C 76, 027302 (2007)

[3] K. Miernik et al., Phys. Rev. Lett. 111, 132502 (2013)

[4] M. Czerwiński et al., Phys. Rev. C 88, 044314 (2013)

[5] T. Materna et al., Phys. Rev. C 92, 034305 (2015)

[6] T. Rząca-Urban et al., Phys. Rev. C 88, 034302 (2013)

[7] E.F. Jones et al., Phys. Rev. C 73, 017301 (2006)

[8] K. Sieja, T.R. Rodríguez, K. Kolos, D. Verney, Phys. Rev. C 88, 034327 (2013)

[9] A. Blanc et al., EPJ Web of Conf. 93, 01015 (2015)

[10] I.N. Gratchev et al., Phys. Rev. C 95, 051302 (2017)

[11] W. Phillips et al., Phys. Lett. B 212, 402 (1988)

[12] https://www.nndc.bnl.gov/ensdf/

[13] S. Chen et al., Phys. Rev. C 95, 041302 (2017) 
[14] M. Lettmann et al., Phys. Rev. C 96, 011301 (2017)

[15] F. Flavigny et al., Phys. Rev. Lett. 118, 242501 (2017)

[16] S. Raman, C.W. Nester, P. Tikkanen, At. Dat. Nucl. Dat. Tab. 78, 1 (2001)

[17] P. Moller, A. Sierk, T. Ichikawa, H. Sagawa, At. Dat. Nucl. Dat. Tab. 109-110, 1 (2016)

[18] J. Litzinger et al., Phys. Rev. C 92, 064322 (2015)

[19] K. Kumar, Phys. Rev. Lett. 28, 249 (1972)

[20] M. Czerwiński et al., Phys. Rev. C 92, 014328 (2015)

[21] D. Rowe, J. Wood, Fundamentals of Nuclear Models: Foundational Models (World Scientific, 2010), ISBN 9789812569554 